\title{
Surface Morphology of Porous Cementitious Materials Subjected to Fast Dynamic Fractures
}

\author{
T. Ficker
}

\begin{abstract}
This paper presents a study of the surface height irregularities of cement pastes subjected to fast dynamic fractures. The height irregularities are quantified by the values of the three-dimensional profile parameters. The studied dynamical irregularities show a similar analytical behavior to those obtained by static fractures.
\end{abstract}

Keywords: 3D profile analysis, fracture surfaces, cement-based materials, confocal microscopy.

\section{Introduction}

We have recently published several studies [1-3] on the surface morphology of fractured specimens made from hydrated cement pastes. This material shows a high value of porosity, which has been proved to be an influential factor governing the irregularities of fracture surfaces.

For practical surface analyses of porous materials, it would be valuable to know whether the surface height irregularities are also influenced by the method of fracture. For this purpose we performed a large series of experiments with cement pastes. This material was chosen because its porosity can easily be controlled within a broad interval by means of the water-to-cement ratio $r=w / c$. Correct knowledge of the dependence of surface roughness on the fracturing method may be useful for further surface studies of fractured porous materials.

\section{Experimental arrangement}

Ordinary Portland cement CEM 42,5 I R-sc of domestic provenance was used to create 144 specimens of hydrated pastes with six different water-to-cement ratios $r(0.3,0.4,0.5,0.6,0.7,0.8)$. The specimens were rotated during hydration to achieve better homogeneity. All specimens were stored for the whole time of hydration at $100 \% \mathrm{RH}$ and $20^{\circ} \mathrm{C}$. After 90 days of hydration the specimens were fractured both in the static regime (three-point bending tests) and also in the dynamic regime (impulse fractures caused by a chisel and a heavy hammer). The fracture surfaces were then immediately used for microscopic analysis.

The three-dimensional profile parameter $H_{a}$ was used to characterize the roughness of the fracture surfaces of the hydrated cement pastes. In fact, $H_{a}$ represents the averaged 'absolute' height of the fracture relief $z=f(x, y)$

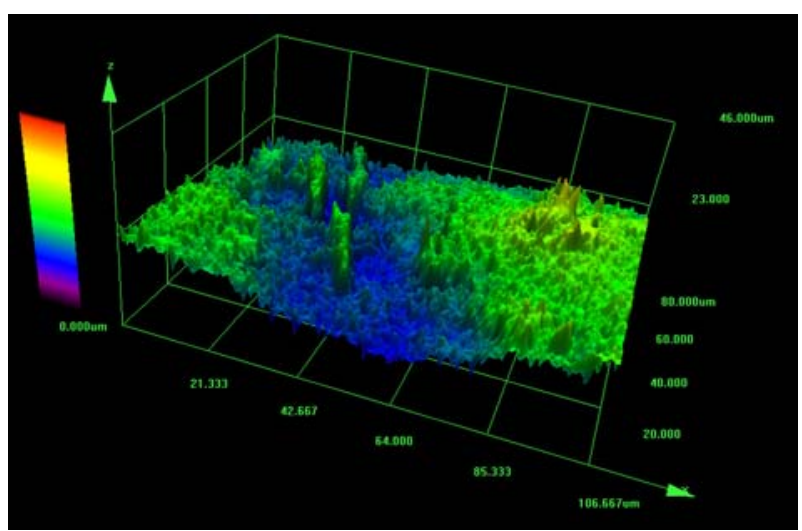

Fig. 1: 3D confocal relief of fractured cement paste

$$
H_{a}=\frac{1}{L \cdot M} \iint_{(L M)}|f(x, y)| \mathrm{d} x \mathrm{~d} y
$$

where $L \times M$ is the area of the vertical projection of the three-dimensional fracture profile $f(x, y)$ into the plane $x y$. The parameter $H_{a}$ has great statistical relevancy, since it is a global averaged characteristic covering the entire tested surface $L \times M$. The three-dimensional profiles $f(x, y)$ were created using an Olympus Lext 3100 confocal microscope. One of these profiles is shown in Figure 1. The profiles are formed by software that processed a series of optical sections created by the confocal microscope at various heights of the fracture surfaces. Approximately 100 image sections were taken for each measured surface site, starting from the very bottom of the surface depressions (valleys) and proceeding to the very top of the surface protrusions (peaks). The investigated area $L \times M=1280 \mu \mathrm{m} \times 1280 \mu \mathrm{m}(1024$ pixels $\times 1024$ pixels) was chosen in five different places of each fracture surface (in the center, and in four positions near the corners of the rectangular area), i.e. each plotted point on the graphs of the profile parameters corresponds to an average value composed 
of 60 measurements $(12$ samples $\times 5$ surface measurements). Each measurement was performed for magnification $20 \times$

\section{Results and discussion}

It is well known that hydrated cement is a composite material consisting of several solid hydrated products and pore spaces (see the photo in Figure 2). Porosity $(P)$ influences most of the mechanical properties of this material, and it is therefore not surprising that the surface irregularity $\left(H_{a}\right)$ was also found $[1-3]$ to be among the dependent properties. Since porosity to a large extent determines compressive strength, a strong functional relation between compressive strength $\sigma_{c}$ and surface irregularity of the fracture surfaces was also revealed

$$
\sigma_{c}\left(H_{a}\right)=\sigma_{o}\left(\frac{H_{o}}{H_{a}-h_{o}}\right)^{\rho}
$$

where $\sigma_{o}, H_{o}, h_{o}$ and $\rho$ are fitting parameters.

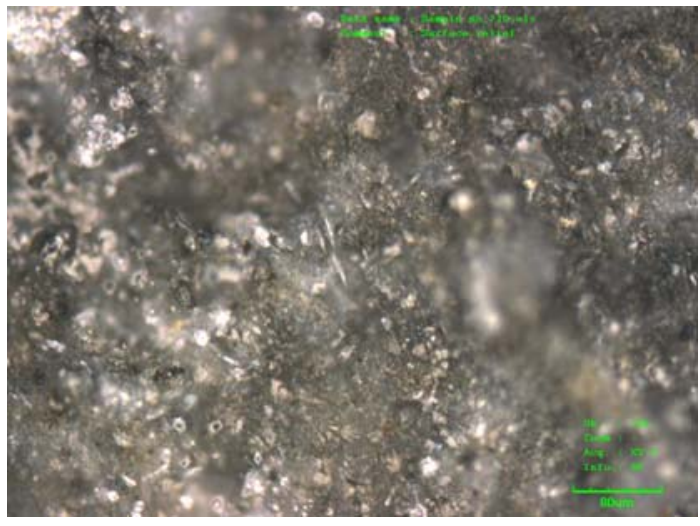

Fig. 2: A photograph of the surface of a hydrated cement paste

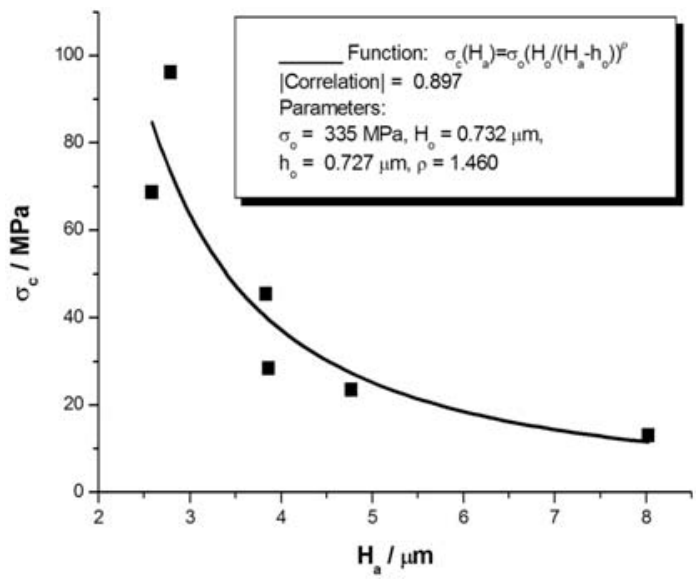

Fig. 3: Compressive strength in dependence on the height irregularities of a surface formed in a dynamic fracture

In the present study, relation (2) is tested with specimens fractured dynamically using a sharp chisel and a heavy hammer that simulate an impulsive load. Figure 3 shows the resulting graph $\sigma_{c}\left(H_{a}\right)$. The dy- namical strength values in Figure 3 were evaluated on the basis of the static values by adding corrections corresponding to dynamic processes [4]. As shown in Figure 3, the experimental points are fitted well by function (2), which means that this function may describe a universal behavior of the compressive strength and the height irregularities of the surfaces formed by both the static fracture processes [3] and the dynamic fracture processes.

\section{Conclusion}

The experiments have proved similar behavior of the height surface irregularities formed by static and dynamic fractures. The fast fracture process accomplished with the wedge-shaped chisel generates very similar graphs $\sigma_{c}\left(H_{a}\right)$ to those in the case of slow fracture processes. These results have been achieved using three-dimensional profile parameters $H_{a}$ evaluated on the basis of a reconstruction of the confocal surface. The graphs $\sigma_{c}\left(H_{a}\right)$ seem to be convenient candidates for calibration curves.

The properties of the surface irregularities of fractured cement pastes presented here may also be useful for morphological and structural studies of other porous materials.

\section{Acknowledgement}

This work was supported by the Ministry of the Czech Republic under Contract no. ME09046 (Kontakt).

\section{References}

[1] Ficker, T., Martišek, D., Jennings, H. M.: Roughness of fracture surfaces and compressive strength of hydrated cement pastes, Cem. Concr. Res. 40 (2010) 947-955.

[2] Ficker, T., Martišek, D., Jennings, H. M.: Surface roughness and porosity of hydrated cement pastes, Acta Polytechnica 51(2011), no. 3, 7-20.

[3] Ficker, T.: Fracture surfaces of porous materials, Acta Polytechnica 51(2011), no. 3, 21-24.

[4] Ficker, T.: Quasi-static compressive strength of cement-based materials, Cem. Concr. Res. 41 (2011) 129-132.

Prof. RNDr. Tomáš Ficker, DrSc.

Phone: +420 541147661

E-mail: ficker.t@fce.vutbr.cz

Department of Physics

Faculty of Civil Engineering

Brno University of Technology

Veveří 95, 66237 Brno, Czech Republic 